\title{
Research on the Obstacle Negotiation Strategy for the Heavy-duty Six- legged Robot based on Force Control
}

\author{
Mantian Li, Enbo Cong, Pengfei Wang and Wei Guo \\ State Key Laboratory of Robotics and Systems, Harbin Institute of Technology, Harbin, 150080, China
}

\begin{abstract}
To make heavy-duty six-legged robots without environment reconstruction system negotiate obstacles after the earthquake successfully, an obstacle negotiation strategy is described in this paper. The reflection strategy is generated by the information of plantar force sensors and Bezier Curve is used to plan trajectory. As the heavy-duty six-legged robot has a large inertia, force controller is necessary to ensure the robot not to lose stability while negotiating obstacles. Impedance control is applied to reduce the impact of collision and active force control is applied to adjust the pose of the robot. The robot can walk through zones that are filled with obstacles automatically because of force control. Finally, the algorithm is verified in a simulation environment.
\end{abstract}

\section{Introduction}

Compared with wheeled robots and tracked robots, legged robots can choose non-continuous foothold, which make them adapt to the rough terrain with obstacles easily [1-2]. Heavy-duty six-legged robots are the most suitable legged robots for transporting supplies because of their characteristic of high stability and strong loading capacity [3]. For heavy-duty robots, the inertia is large, force control is extremely necessary. If the robot lose stability while contacting obstacles, it will be difficult to adjust back. So the pose of the robot need to be adjusted in real time.

At present, there are two methods for muti-legged robots to negotiate obstacles. One is recognising obstacles by environment reconstruction devices. For instance, the Little Dog built a 3D terrain model and picked up obstacle information by vision sensor, then they planned an appropriate path to negotiate obstacles [4]. But this method will be restricted by the environmental factors. Sandstorm and rain-snow environment will affect the performance of the vision sensor, laser radar or other external environment sensors. So this method can't be applied to the robot working in the severe environment. The other method is receiving contact information by plantar force sensors. Typical examples include Tekken designed by Japanese [5] and DLR-Crawler designed by German [6]. These robots replanned the trajectory after contacting obstacles. As soon as plantar force sensors receive signals that any foot has contacted obstacles, robots adjust swing track quickly. Central pattern generator(CPG) controller is used in Tekken and the distributed artificial neural network controller WALKNET is used in DLR-Crawler. All the two intelligent control technology need large computational quantity and not handy for real-time control.

This paper describes a strategy for the heavy-duty sixlegged robot to negotiate obstacles in real-time in the severe environment. Reflection generation strategy and force control strategy are presented in detail in this paper.

The rest sections are organized as follows. In Section 2 , the model is established. Sections 3 and 4 describe the two subtasks of obstacle negotiation strategy for heavyduty six-legged robot: the reflection strategy and the force control strategy. Section 5 presents results of simulation. Finally, Section 6 summarizes the work and presents the conclusions that can be drawn from it.

\section{Model}

A model in Adams is established to simulate the real environment, as we can see in Figure 1.

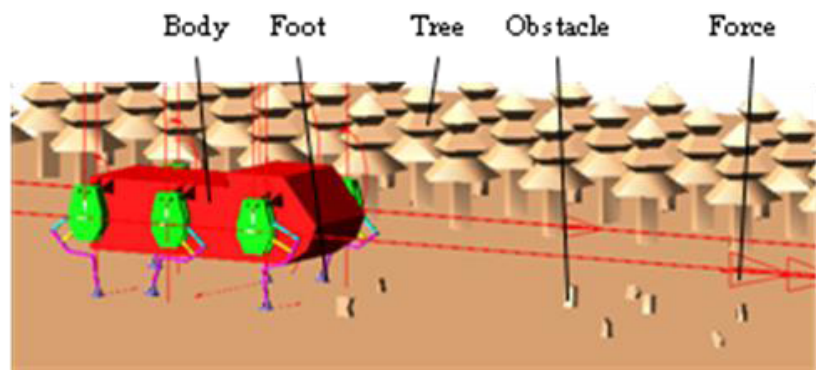

Figure 1. Model in Adams.

The model's parameters are shown in Table 1,where $M$ represents the weight of the heavy-duty six-legged robot, $H$ represents the height from bottom of the body of the robot to ground. $L$ represents the length of the robot. $d$ represents the width of the robot. $f$ represents friction 
factor between feet of the robot and ground. $n$ represents joints number in one leg. $h_{\max }$ represents the vertical height of the highest obstacle.

Table 1. Parameters of the model.

\begin{tabular}{ccccccc}
\hline$M$ & $H$ & $L$ & $d$ & $f$ & $n$ & $h_{\max }$ \\
\hline 3000 & 1000 & 4000 & 2200 & 0.5 & 3 & 350 \\
$\mathrm{~kg}$ & $\mathrm{~mm}$ & $\mathrm{~mm}$ & $\mathrm{~mm}$ & & & $\mathrm{~mm}$ \\
\hline
\end{tabular}

\section{Reflection Strategy}

\subsection{Establishment of reflection rule}

Legs may contact obstacles at any time in a period time, different reflection rules are made for different collision time. We can insert 4 time points $\mathrm{A} \sim \mathrm{E}$ during the swing phase time, which divide the swing phase into 3 stages, as shown in Figure 2.
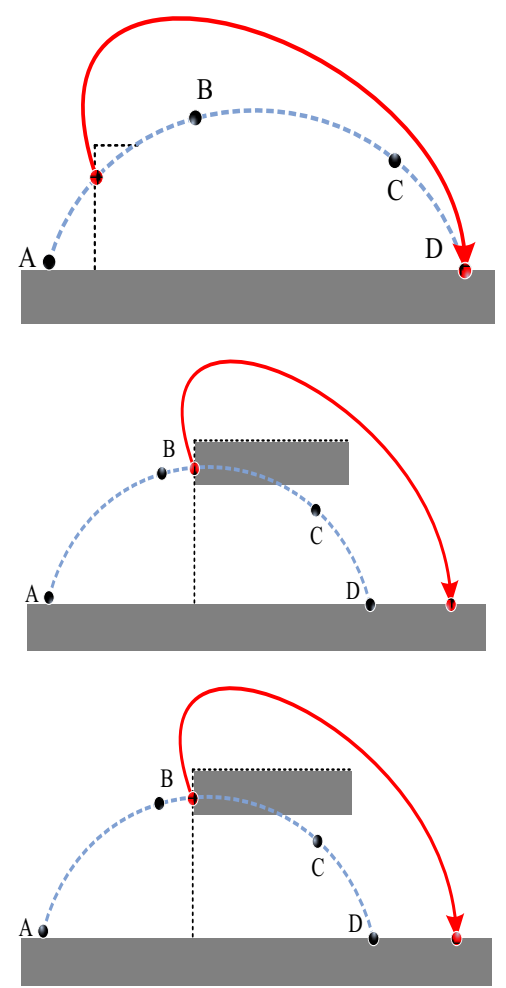

Figure 2. Different reflection rule in different situations.

In Figure 2, $\mathrm{AB}$ is the early stage of the swing motion. If the foot contacts obstacles at this stage, reflection motion will be trigged. The foot will be retreated and the height of the new trajectory is higher than the original trajectory.

$\mathrm{BC}$ is the later stage, and the reflection rule is roughly identical to $\mathrm{BC}$. The difference is that the foothold of $\mathrm{BC}$ is in front of the foothold of AB.

$\mathrm{CD}$ is the final stage of the swing motion. The foot will be retreated to a position near the obstacles if touching obstacles because the time left is not enough for the foot to accomplish negotiating obstacles.

As stated above, reflection rule is established based on collision time, then it can ensure the foot to have enough time to negotiate obstacles.

\subsection{The curve of reflection Trajectory}

For the heavy-duty six-legged robot, the trajectory need to be smooth and compliant. So Bezier Curve is applied to plan the trajectory, because the main advantage of this kind of curve is smooth and compliant. Parametric equation of $\mathrm{n}$ order Bessel curve is described as $Q(t)$ in Eq.(1).

$$
Q(t)=\sum_{i=0}^{n} P_{i} B_{i, n}(t) \quad t \in[0,1]
$$

Where $P_{i}$ represents position vector of $\mathrm{n}+1$ control points. $B_{i, n}(t)$ represents Bernstein polynomial, which can be described in Eq.(2).

$$
B_{i, n}(t)=\frac{n !}{i !(n-i) !} t^{i}(1-t)^{n-i}
$$

If $t_{\text {swing }}$ represents the total time of swing phase, $t_{\text {collision }}$ represents the collision time point, the terminal velocity $v_{s f}$ of the foot in swing phase can be calculated by Eq.(1) and Eq.(2), as shown in Eq.(3).

$$
v_{s f}=\dot{Q}(1)=n\left(P_{n}-P_{n-1}\right) \frac{1}{t_{\text {swing }}-t_{\text {collision }}}
$$

In Eq.(3), if appropriate values are assigned to $P_{n}$ and $P_{n-1}$, the terminal velocity of foot in swing phase will equal to the velocity of foot in stance phase. In this way, the sudden change of velocity can be eliminated. Then the trajectory will be smooth enough for heave-duty robot to negotiate obstacles.

\section{Force Control Strategy to Guarantee Stability}

To guarantee stability of the heavy-duty robot, force control strategy is applied. Impedance control strategy is applied to swing legs, and active force control strategy is applied to stance legs. These two strategies are described as follow.

\subsection{Impedance control strategy for swing legs}

Compared with light-duty robots, heavy-duty robots have large inertia. The impact force caused by the collision between robot and convex obstacles is large enough to make the robot overturn. So in order to reduce the impact of collision, position-based impedance control method is applied to swing legs.

Impedance control is a method to adjust force and position dynamically. The input of impedance controller is the deviation $\Delta F$ between actual contact force $F_{\gamma}$ measured by plantar force sensors and target force $F^{\prime}$. It's obvious $F^{\prime}=0$ in the swing phase. The output of impedance controller is the deviation $\Delta P$, which can adjust the current position $P_{\gamma}$ to a position $P$ where $\mathrm{F}^{\prime}=0$, as shown in Figure 3. 


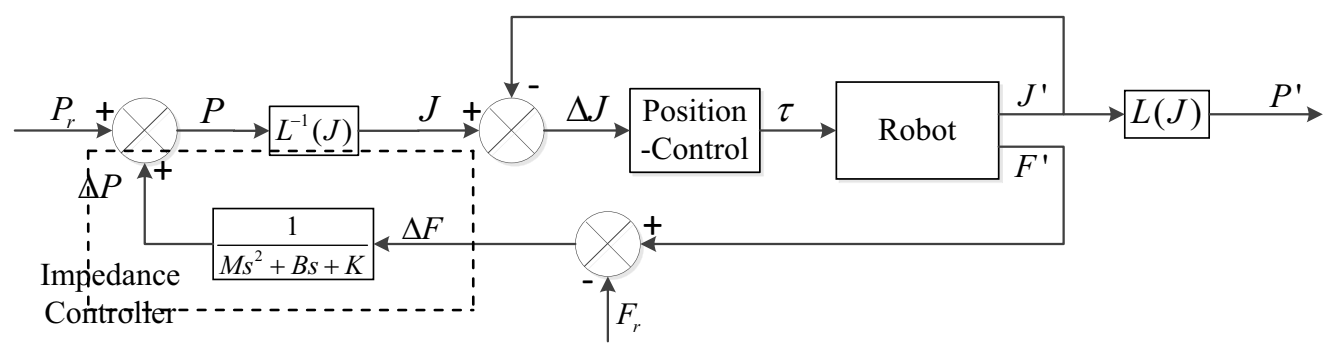

Figure 3. Impedance control system for swing legs.

Impedance controller can be described as Eq.(4).

$$
\Delta F=M_{d} \Delta \ddot{P}+C_{d} \Delta \dot{P}+K_{d} \Delta P
$$

Where $M_{d}$ represents inertia coefficient, $C_{d}$ represents damping coefficient and $K_{d}$ represents stiffness coefficient.

By impedance controller, contact force and position of the foot are dynamically adjusted. If appropriate $M_{d}$, $C_{d}$ and $K_{d}$ are assigned to the controller, the foot can get away from obstacles quickly.

\subsection{Active force control strategy for stance legs}

When the robot contact obstacles, there will be an error between actual pose and target pose of the body caused by collision. The greater the impact force is, the larger the error is. Large error may cause the robot lose its stability. So the pose is adjusted through allocating appropriate force to every stance foot.

The pose of the robot is adjusted by virtual suspension model, which is an imaginary spring-damping system. As shown in Figure 4.

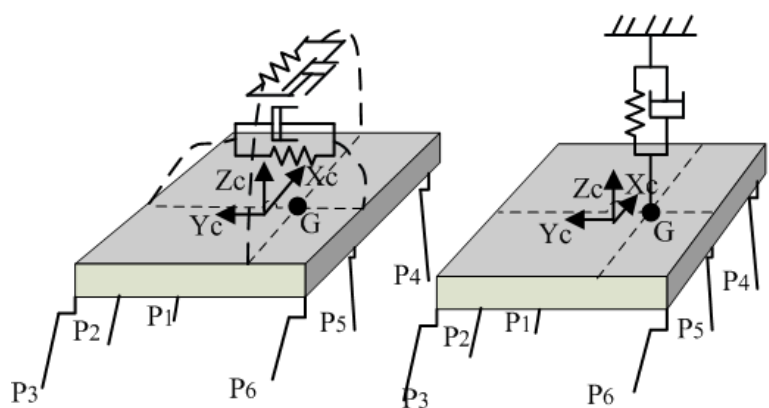

Figure 4. Three DOFs of virtual suspension model.

In Figure 4, virtual suspension model is used to adjust pitch angle, roll angle and vertical height of the robot. The pose of robot is measured by inertial navigation devices in real-time. When the robot contacts obstacles, there will be deviations between actual pose and target pose. If the deviation of pitch angle is represented by $\Delta \beta$, the deviation of roll angle is represented by $\Delta \gamma$, and the deviation of vertical height is represented by $\Delta d$, there will be corresponding virtual generalized force $\Delta M_{\beta}, \Delta M_{\gamma}$, $\Delta M_{d}$ provided by stance legs.

The virtual generalized force required to eliminate deviation is shown as Eq.(5). Stiffness coefficients $k_{\beta}, k_{\gamma}$, $k_{d}$ and impedance coefficients $c_{\beta}, c_{\gamma}, c_{d}$ are parameters to correct the deviations.

$$
\begin{aligned}
& \Delta M_{\beta}=k_{\beta} \Delta \beta-c_{\beta} \Delta \dot{\beta} \\
& \Delta M_{\gamma}=k_{\gamma} \Delta \gamma-c_{\gamma} \Delta \dot{\gamma} \\
& \Delta F_{d}=k_{d} \Delta d-c_{d} \Delta \dot{d}
\end{aligned}
$$

In fact, $k_{\beta}, k_{\gamma}, k_{d}$ are related to control stiffness in $z$ direction of stance legs, which is represented by $K_{i z}$. Their mathematical relationship is shown as Eq.(6).

$$
\begin{aligned}
k_{\beta}= & \frac{\sum_{i=1}^{6} K_{i z}\left({ }^{C} P_{i x}-{ }^{C} G_{x}\right) \sin \Delta \beta\left({ }^{C} P_{i x}-{ }^{C} G_{x}\right)}{\Delta \beta} \\
\approx & \sum_{i=1}^{6} K_{i z}\left({ }^{C} P_{i x}-{ }^{C} G_{x}\right)^{2} \\
k_{\gamma}= & \sum_{i=1}^{6} K_{i z}\left({ }^{C} P_{i y}-{ }^{C} G_{y}\right) \sin \Delta \gamma\left({ }^{C} P_{i y}-{ }^{C} G_{y}\right) \\
& \approx \sum_{i=1}^{6} K_{i z}\left({ }^{C} P_{i y}-{ }^{C} G_{y}\right)^{2} \\
k_{d}= & \frac{\sum_{i=1}^{6} K_{i z} \Delta d}{\Delta d}=\sum_{i=1}^{6} K_{i z}
\end{aligned}
$$

Where $i=1,2, \cdots, 6 .{ }^{C} P_{i x}$ represents $i$ th foot position along $x$ coordinate in the body coordinate system, ${ }^{C} P_{i y}$ represents $i$ th foot position along $y$ coordinate in the body coordinate system. ${ }^{C} G_{x}$ represents centre of gravity position along $x$ coordinate in the body coordinate system. ${ }^{C} G_{i y}$ represents centre of gravity position along $y$ coordinate in the body coordinate system.

By Eq.(5) and Eq.(6) and coordinate transformation equation, we can calculate position variation of every foot relative to the body, as shown in Eq.(7).

$$
\Delta{ }^{C} P_{i z}=-{ }^{C} P_{i x} \frac{\Delta M_{\beta}}{k_{\beta}}+{ }^{C} P_{i y} \frac{\Delta M_{\gamma}}{k_{\gamma}}+\frac{\Delta F_{d}}{k_{d}}
$$

Then the force ${ }^{C} F_{i z}(i=1,2,3,4,5,6)$ allocated to every foot to eliminate the variation can be calculated by Eq.(8).

$$
{ }^{C} F_{i z}=-\left(K_{i z}{ }^{C} P_{i x}\right) \frac{\Delta M_{\beta}}{k_{\beta}}+\left(K_{i z}{ }^{C} P_{i y}\right) \frac{\Delta M_{\gamma}}{k_{\gamma}}+\left(K_{i z}\right) \frac{\Delta F_{d}}{k_{d}}
$$

By allocating force, the robot can walk stably when it contacts convex obstacles. 


\section{Simulation and Analysis}

As trajectory planning and force control for negotiating obstacles have been completed, the algorithm is verified by joint simulation of Simulink and Adams, the sample period of which is $10 \mathrm{~ms}$, as shown in Figure 5.

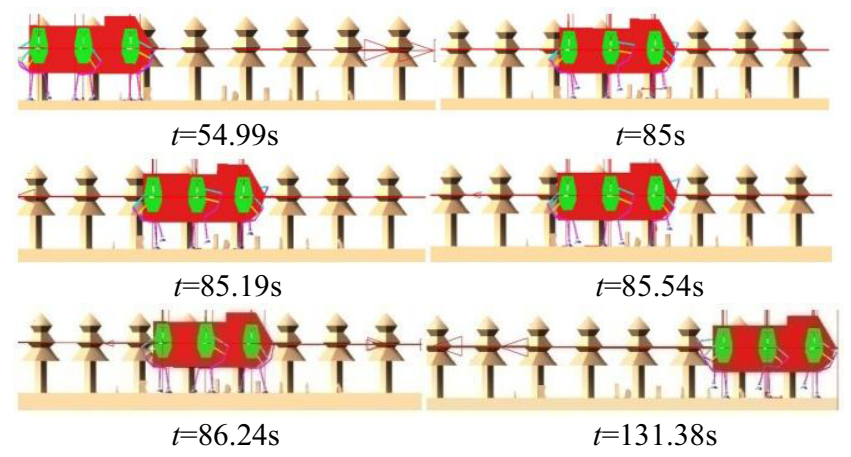

Figure 5. Process of obstacle negotiation in simulation.

In Figure 5, the robot is able to pass through terrain with plenty of convex obstacles after thefiftieth second. Figure 6 is the trajectory when a foot contact obstacles 3 times in a swing period. And the trajectory generated by Bezier Curves is smooth enough to ensure the robot to negotiate obstacles.

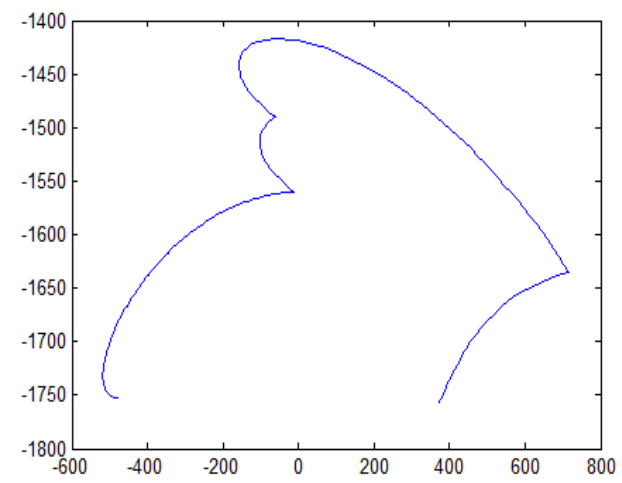

Figure 6. Trajectory generated by Bezier Curves.

As force control strategy has been applied, the roll angle, pitch angle and height of the robot can be maintained around target values, which is shown in Figure 7. The deviations between target values and actual values are so small that can be ignored.

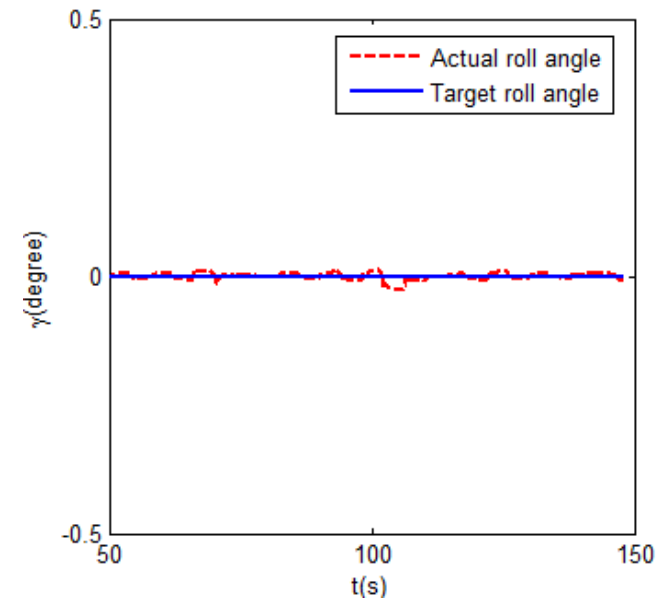

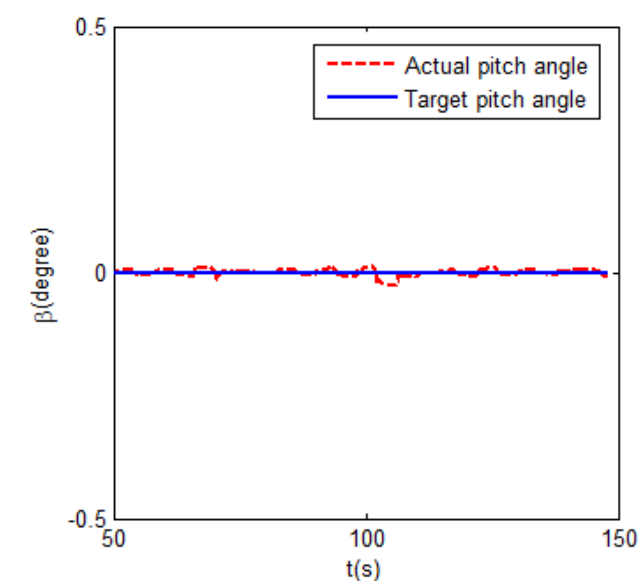

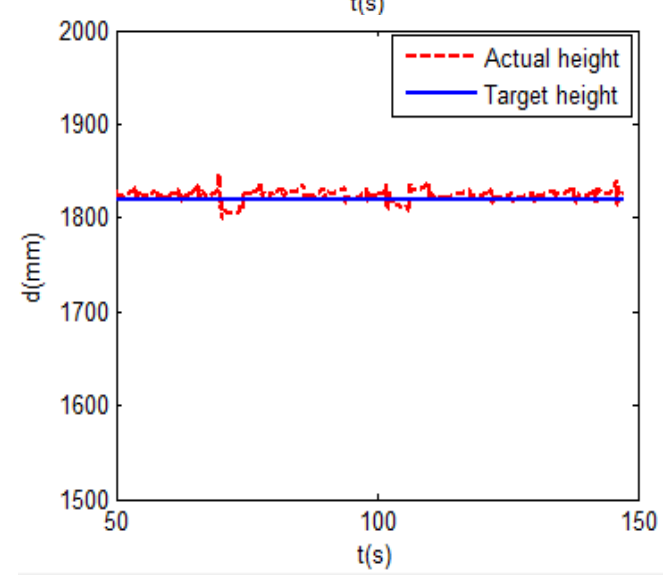

Figure 7. Results of maintaining pose during the collision.

\section{Conclusion}

An obstacle negotiation strategy for heavy-duty sixlegged robot without environment reconstruction system is described in this paper. A trajectory planning strategy is established by the use of plantar force sensors and Bezier Curves is used to plan trajectory. In order to prevent heavy-duty six-legged robots with large inertia from overturning, impedance control strategy is applied to swing leg, and active force control is applied to stance legs. Finally, the algorithm is verified to be correct and effective by joint simulation.

\section{References}

1. R.B. McGhee, G.I. Iswandhi, IEEE Trans Syst Man Cybern A 9,176(1979)

2. Q.J.Huang,K.Nonami, ASME T MECH 13,773(2003)

3. E. García Armada, M. A. Jiménez, G.D.S Pablo, IEEE Trans Rob Autom 14,90(2007).

4. V. Dürr, J. Schmitz, H. Cruse, Arthropod Struct DEV 33, 37(2004)

5. S. M. Song, K. J. Waldron, Nasa Sti/Recon Technical Report A, 89, 7 (1989)

6. J. Z. Kolter, M. P. Rodgers, A. Y. Ng., International Conference on Robotics and Automation(IEEE, New York, 2015) 